\title{
Combined Pediatric and Adult Trials Submitted to the US Food and Drug Administration 2012-2018
}

\author{
Irin Tanaudommongkon ${ }^{1}$, Shogo John Miyagi ${ }^{2}$, Dionna J. Green ${ }^{3}$, Janelle M. Burnham ${ }^{1}$, \\ John N. van den Anker ${ }^{2}$, Kyunghun Park ${ }^{1}$, Johanna $\mathrm{Wu}^{4}$, Susan K. McCune ${ }^{3}$, Lynne $\mathrm{Yao}^{5}$, \\ Gilbert J. Burckart ${ }^{1}$ \\ ${ }^{1}$ Office of Clinical Pharmacology, Center for Drug Evaluation and Research, US Food and Drug \\ Administration, Silver Spring, MD \\ ${ }^{2}$ Division of Clinical Pharmacology, Children's National Hospital, Washington, DC \\ ${ }^{3}$ Office of Pediatric Therapeutics, Office of the Commissioner, US Food and Drug Administration, \\ Silver Spring, MD \\ ${ }^{4}$ Western University of Health Sciences, Pomona, CA \\ ${ }^{5}$ Division of Pediatric and Maternal Health, Office of New Drugs, Center for Drug Evaluation and \\ Research, US Food and Drug Administration, Silver Spring, MD.
}

\section{Abstract}

Despite legislation incentivizing and requiring drug companies to conduct pediatric clinical trials, there still is a 9-year delay in drug approval for pediatric labeling after the initial adult drug approval. The aim of this study was to review the experience of the US Food \& Drug Administration (FDA) with combined pediatric and adult trials as a means for expediting pediatric approval and labeling. Combined pediatric and adult trials submitted to the FDA from 2012 to 2018 were reviewed. Only the publicly available labels and reviews were utilized for this analysis. Combined trials were identified for 72 products, with a total of 156 combined adult and pediatric trials. The therapeutic areas with the largest number of combined trials were in pulmonology for products reviewed under the Best Pharmaceuticals for Children Act (BPCA) and/or the Pediatric Research Equity Act (PREA), and hematology reviewed under the Orphan Drug Act (ODA). All drugs that utilized combined pediatric and adult clinical trials were approved simultaneously for both the adults and that part of the pediatric population. A separate pediatric subgroup efficacy analysis was reported in 57\% and $48 \%$ of products under BPCA/PREA and the ODA, respectively, with a separate safety analysis in $48 \%$ and $38 \%$ of these products. When considering both BPCA/

Correspondence: Gilbert J. Burckart (Gilbert.Burckart@fda.hhs.gov) Office of Clinical Pharmacology, U.S. Food and Drug Administration, 10903 New Hampshire Avenue Building 51-3184, Silver Spring, MD 20993. Telephone 301-796-2065.

Author Contributions

Wrote the manuscript: IT, SJM, DJG, JMB, JNVDA, KP, JW, SKM, LY, GJB

Designed the research: IT, SJM, DJG, KP, GJB

Performed the research: IT, SJM, KP, JW, GJB

Analyzed the data: IT, SJM, KP, JW, GJB

Conflict of Interest: All authors declared no competing interests for this work.

Conference presentation: None

Publisher's Disclaimer: Disclaimer: The opinions expressed are those of the authors and should not be interpreted as the position of the US Food and Drug Administration. 
PREA and orphan drug studies, all the combined pediatric and adult trials allowed concurrent approval and labeling for part of the pediatric population at the time of the adult approval.

\section{Keywords}

combined pediatric-adult trials; pediatric; orphan drugs; BPCA; PREA

\section{Introduction:}

Medications are frequently used off-label in pediatric patients. ${ }^{1}$ Approximately 62 to $85 \%$ of drugs prescribed for children are used off-label, ${ }^{2}$ with higher rates for children hospitalized in neonatal and pediatric intensive care units. ${ }^{3}$ While off-label use does not imply improper, contraindicated, or illegal use, off-label use often lacks substantial evidence for efficacy in contrast to that required for approval. The lack of pediatric use information for approved drugs is also associated with an increased risk for adverse drug reactions. ${ }^{4}$ Pediatric healthcare providers are then faced with the practical dilemma as to how to prescribe offlabel medications for children due to a lack of reliable information.

Drug development in pediatric patients is often complicated by challenges such as ethical and legal aspects, appropriate pediatric formulations, small sample sizes, and slow recruitment into trials. ${ }^{5}$ Prior to the passage of the Best Pharmaceuticals for Children Act (BPCA) in 2002 and the Pediatric Research Equity Act (PREA) in 2003, few pediatric drug product trials were submitted to the FDA. FDA has the authority to require pediatric studies under certain circumstances (PREA), and also to incentivize drug developers to conduct pediatric studies (BPCA). These laws were reauthorized, made permanent, and further strengthened through the passage of the FDA Amendments Act (FDAAA, 2007), the FDA Safety and Innovation Act (FDASIA, 2012), and the FDA Reauthorization Act (FDARA, 2017).

PREA and BPCA have contributed to the increase in pediatric trials submitted to the FDA and subsequently approved pediatric indications, ${ }^{6-8}$ but many drug labels still lack pediatric information. ${ }^{2}$ Also, many pediatric trials required under PREA are deferred as postmarketing requirements (PMRs). As of January 2019, there were 182 PMRs in pending status, denoting that the pediatric trials have not yet been completed. ${ }^{9}$ The completion rate of pediatric PMRs after product approval was $2.3 \%$ after one year, $6.8 \%$ after two years, and reached $26.7 \%$ five years after approval. ${ }^{2}$ Since the product is available for use in pediatric patients after adult approval, it may be more difficult to enroll children in clinical trials as the time after adult approval increases.

The Orphan Drug Act (ODA) of 1983 was enacted to facilitate the approval of drugs for rare diseases, defined as diseases that affect less than 200,000 individuals in the U.S. Under the ODA, sponsors may receive an additional seven years of marketing exclusivity for the approval of an indication that has received orphan designation. Products with orphan designations are exempt from PREA requirements, which could prevent the conduct of pediatric studies that might normally be required under PREA. ${ }^{10}$ Between 1999 and 2018, FDA identified 548 orphan indications that were approved during the review period, with 
200 orphan indications that did not warrant pediatric labeling. ${ }^{11}$ Of the total of 548 orphan indications, FDA deemed 348 indications (64\%) to be relevant to children, thus warranting pediatric use information in the labeling for the indication. Of these 348 indications, 221 (64\%) indications for which pediatric use information was considered to be appropriate were fully labeled (i.e., the labeling contains adequate efficacy, safety, dosing, and ageappropriate formulation information to support its use in the full range of affected pediatric patients) for pediatric use. FDA identified 127 orphan indications that were not fully labeled for the pediatric population. The 127 orphan indications missing pediatric information in labeling corresponded to 98 drugs approved for one or more orphan indications ( 19 of the 98 drugs were approved for more than one orphan indication). Approximately two-thirds (81) of these indications had no pediatric information at all, while the remainder had some pediatric labeling. ${ }^{11}$

One method of improving the timeline for pediatric drug approval and labeling is through the concurrent inclusion of pediatric and adult participants in drug development studies. Combined pediatric and adult trials allow for the simultaneous evaluation of safety and efficacy, particularly during Phase 3 studies, and has the advantage of obtaining approval in part of the pediatric population at the time of the adult drug approval. Collated experience over time with conducting pediatric trials, increased understanding in the similarities and differences of certain diseases and response to therapy between adults and pediatrics, and advances in deriving pediatric dosing support the rationale for inclusion of pediatric populations in adult pivotal trials, when appropriate. The FDA has encouraged this approach, and recently published FDA draft guidances discuss the inclusion of adolescent patients in adult clinical trials. ${ }^{12,13}$

Numerous challenges exist when designing and implementing a combined pediatric and adult trial and highlight the fact that a combined trial approach may not be suitable for every disease indication or scenario. In general when considering a combined trial approach for a given indication, there should be an expectation that similar disease progression occurs in both children and adults, and that a similar exposure-response relationship exists for children and adults. ${ }^{14}$ These expectations are the same as occurs in patient recruitment in any drug development study. However, even when this is the case, other factors may make a combined adult and pediatric trial challenging to design and implement. For example, different primary endpoints measured in adult trials for a given indication may not always be suitable for use in pediatric trials. ${ }^{15}$ In addition, the need to enroll sufficient numbers of pediatric patients for safety and efficacy evaluation and the small numbers of pediatric patients available also make subgroup analyses difficult to interpret. Adolescents may the most feasible pediatric population to enroll in combined trials, but developmental toxicities such as impairment in growth or impact on sexual maturation can preclude combined trials. ${ }^{16}$

Given the potential for increasing the rate of pediatric approvals in at least part of the pediatric population at the time of adult drug approvals, the experience of the FDA with combined pediatric and adult trials is particularly valuable. The objectives of this evaluation were 1) to perform a review of the BPCA and/or PREA and orphan drug submissions to the FDA where combined adult and pediatric trials were conducted, 2) to evaluate the most relevant therapeutic areas in which combined trials were conducted, 3) to identify the trials 
that reported separate safety and efficacy analyses for the pediatric subgroups, and 4) to delineate which dosing methods were most often utilized in the combined trials.

\section{Methods}

\section{Data Sources}

The drugs were categorized as either (i) BPCA and/or PREA or (ii) ODA and were then subcategorized into either drug or biologic products. Orphan drug submissions are exempt from PREA, but BPCA can also pertain to orphan drugs.

All drug development programs submitted to the FDA under the ODA (January 1, 2012 to December 31, 2018) or under BPCA/PREA (July 1, 2012 to December 31, 2018) were surveyed. These studies were conducted under FDASIA (July 2012 to July 2017), FDARA (August 2017 to December 2018) and the ODA. These dates were chosen as being contemporaneous with current drug development practices. Data were extracted from the publicly available FDA approved labels, approval letters, and authored FDA reviews for each trial, including cross-team leader reviews, medical reviews, clinical pharmacology reviews, and statistical reviews. Additional information regarding clinical studies was also retrieved from ClinicalTrials.gov and journal publications indexed on PubMed/Medline. This study did not require Research Involving Human Subjects Committee review and approval under 45 CFR $\$ 46.101 b(4)$.

\section{Definitions and Outcomes}

Drugs were classified into the following categories according to the primary indication or affected organ system: allergy, anti-infectives, antiviral, dermatology, hematology, metabolic, neurology, oncology, ophthalmology, psychiatry, gastroenterology, rheumatology, inborn errors of metabolism or pulmonology. Age groups were defined as: pediatrics/ adolescents ( $<18$ years) ${ }^{17}$ and adults ( $\geq 18$ years). For dosing, fixed dosing refers to dosing strategies without correction for body size. Weight-based dosing is calculated based on an individual's body weight, while weight band dosing refers to using cutoffs on certain doses based on a weight range.

\section{Data Collection}

Data were collected from publicly-available FDA sources. If the clinical trial information was incomplete, additional information was obtained through ClinicalTrials.gov or PubMed/ Medline if available. Information regarding each drug or biologic submission was collected and included the following: therapeutic area, legislation under which the study took place (i.e. BPCA, PREA, or ODA), original label indication (if already approved), previous indication before submission (if multiple submissions existed), intended indication for submission, approved indication for submission, formulation, drug class and whether the drug was a new-molecular entity (NME), and number of trials included in the submission. The following information regarding each drug trial was collected: clinical trial design, trial phase, trial outcome, inclusion criteria age, actual age range enrolled in study, duration of study, number of total subjects, total number of pediatric subjects divided into age groups, 
dose(s) and dose selection process, subgroup efficacy analysis, subgroup safety analysis, subgroup age stratification, and pooled safety analysis.

To determine whether additional pediatric trials beyond the combined adult and pediatric trial were necessary, both labels and a listing of postmarketing requirements and commitments were examined.

\section{Statistical Analysis}

Standard summary statistics including counts were used to describe the study variables. The outcomes for this analysis were therapeutic area, years between initial approval and labeling for pediatric use, year approved for pediatric use, and fixed dose/weight band/weight-based dosing. Descriptive analyses were performed regarding the safety, efficacy, dosing type, and therapeutic area.

\section{Results}

There were 72 total drug and biological products and 156 trials submitted to the FDA that were identified as having combined pediatric and adult trials, based on this systematic review of drug and biological products from 2012 to 2018. The majority of these combined trials included the entire pediatric age range that would benefit from the treatment ( 91 trials for 42 products). A substantial number included only adolescents (12-18 years of age; 56 trials in 24 products) whereas some programs included older children and adolescents (618 years of age; 9 trials in 6 products). A more complete breakdown of ages in BPCA/PREA or under the ODA is presented in Table 1.

\section{Drugs Approved under PREA and BPCA}

Drug Products-Forty-six trials were conducted on 23 products under BPCA and/or PREA for drug products. The most commonly studied therapeutic area under BPCA and/or PREA for drug products was pulmonology, which consisted of 33\% (15/46) of the total studies (Figure S1a) and 22\% (5/23) of the total drug products (Figure 1a). The pediatric population included in the combined trial was approved concurrently with the adult population in all cases.

The number of combined trial approvals by year are shown in Figure 2. There was an average of three drugs approved per year from 2012 to 2017 (Figure 2a) using the combined trials approach. For efficacy, 56.5\% (26/46) of trials performed a separate efficacy analysis for the pediatric patients (Table 2). For safety, 46\% (21/46) of trials reported a separate safety analysis for pediatric patients (Table 2). Fixed dosing was utilized in 83\% (38/46) of the studies (Table 3), while 11\% (5/46) used weigh-based dosing and 6.5\% (3/46) used weight band dosing.

Biological Products-There were four biological products approved under BPCA and/or PREA in the combined trials. Pulmonology $(75 \%)$ and allergy (25\%) were the only two therapeutic areas for these combined trials. All products received simultaneous FDAapproval in adults and for the pediatric population included in the combined trial for the indication studied. Figure 2a shows that there was one biologic approved each year from 
2014 to 2017 using combined pediatric and adult trials. All the studies were safety and efficacy studies, and 62.5\% (5/8) of the studies performed a separate efficacy and safety analysis for the pediatric subgroup. Only one product performed a combined adult and pediatric dose-finding study. Fixed dosing for biological products was used in $87.5 \%$ (7/8) of products, and one product used weight-based dosing.

\section{Drugs approved under the ODA}

In this analysis, a total of 45 drug and biological products were approved from 2012 to 2018 under the ODA, utilizing a total of 102 combined pediatric and adult trials. Of this group of orphan drug trials, $54 \%$ (55/102) were for biological products and $46 \%(47 / 102)$ were for drug products.

Drug Products-The orphan approvals for drug products are in ten therapeutic areas but are concentrated in two therapeutic categories: hematology 19\% (9/47) and neurology 19\% (9/47) based on the number of trials (Figure S1c). When evaluating the drug approvals by the number of drug products, hematology $20 \%$ (4/20) was the most common therapeutic area (Figure 1c). All of drug products were approved simultaneously for pediatric use for the population studied along with the adult drug approval. For efficacy, 60\% (28/47) of these studies performed a separate efficacy analysis in the pediatric patients. For safety, $45 \%$ (21/47) of combined safety trials evaluated safety in the pediatric patients separately. A few drugs had overlapping dosing regimens (e.g., fixed dose and weight band), but most studies (45\%) (21/47) used weight-based dosing. Only one (1/47) of the studies was a dose-finding study (Table 2).

Biological Products-Most orphan approvals for biological products were hematology studies $44 \%$ (24/55) as the major therapeutic area, follow by oncology $18 \%$ (10/55). No trend was observed between the number of biological products and the year approved for pediatric use on the basis of combined trials for the 2012 to 2018 period. The concurrent approval in the pediatric patients included in the combined trials was observed for biological products at the time of the adult approval.

Weight-based dosing was used in $84 \%$ (46/55) of the studies, and there were about equal numbers of fixed dosing and weight band dosing of around 30\%. A separate efficacy evaluation for the pediatric subgroup was documented in 38\% (21/55) of the total studies for biological products. For safety endpoints, 33\% (18/55) of combined trials performed a separate safety evaluation for the pediatric subgroup. Only $4 / 55$ studies performed was a dose finding study.

\section{Discussion}

Pediatric approval is often delayed in the drug development process. As a consequence, pediatric drug and biological product approvals lag behind adult approvals by an average of nine years. This time lag can contribute to difficulty in completing pediatric drug development trials. Conducting combined pediatric and adult combined trials is one solution to this significant time delay in pediatric approval. ${ }^{18}$ 
This is not the first attempt to expedite pediatric drug development programs. The extrapolation of efficacy data from adults to pediatric patients was included in the final regulation on pediatric labeling in 1994. With extrapolation of efficacy from adults to pediatric patients, the pediatric development program is reduced to dose determination and safety studies. Support for this concept was reaffirmed in 2019 in the draft US FDA Guidance for Industry: Demonstrating Substantial Evidence of Effectiveness for Human Drug and Biological Products, ${ }^{14}$ and extends to the 2018 EMA "Reflection paper on the use of extrapolation in the development of medicines for paediatrics." 19 A review of these US pediatric extrapolation programs in $2017^{20}$ demonstrated changing ideas about the assessment of disease similarity between adults and pediatric patients, and these concepts will continue to evolve.

For the approval of a drug or biological product in the United States, the FDA requires substantial evidence of effectiveness. ${ }^{14}$ Combined adolescent-adult trials however are challenging in the pediatric population for a number of reasons, including ethical concerns with differing requirements between adults and adolescents for assent/consent, ${ }^{21}$ psychosocial issues for adolescents, ${ }^{22}$ recruitment challenges when adolescents may be cared for at different sites from adults, and the potential delay in completion of the phase 3 trials if these considerations become obstructions. The proposed mitigation strategies for these challenges include: (a) early discussions with the review division at the FDA, (b) the fact that no minimum number of adolescents is required, (c) selecting study sites with both adults and adolescents, (d) selecting sites where institutional review boards cover both adults and adolescents, (e) including pediatric experts on safety committees and as principal coinvestigators, and (f) including adolescents in community discussions of trial design and ethical approval. ${ }^{23}$ Support for these trials has come from both the position paper of the American College of Clinical Pharmacology and the Institute for Advanced Clinical Trials for Children (I-ACT) conference on "The Inclusion of Adolescents in Adult Clinical Trials" in October, 2019.

The FDA position in encouraging these combined adult and pediatric trials is demonstrated in the release of specific draft guidances in the oncology and in antiviral products. In the past, "pediatric trials of the same drug generally have been initiated after the completion of one or more adult clinical trials, or after the initial approval in adults, delaying development of and access to potentially effective new cancer drugs for the pediatric population." 12 To address this issue, FDA released the draft 2019 Guidance for Industry: Considerations for the inclusion of adolescent patients in adult oncology clinical trials. ${ }^{12}$ The purpose of the guidance is to enable earlier access to drug products for adolescent patients with cancer. Adolescent patients should be eligible for participation in adult oncology trials in all stages of drug development when the tumor histology and biologic behavior is the same.

Adolescent patients may be enrolled after obtaining initial adult pharmacokinetic and toxicity data. Additionally, for dose escalation for drugs with body size-adjusted dosing for adults, "adolescent patients should receive the same body size-adjusted dose ( $\mathrm{mg} / \mathrm{kg}$ or $\mathrm{mg} / \mathrm{m}^{2}$ or other suitable binning) that is administered in adults." Safety monitoring data in such a trial should also be examined for any age-related differences. ${ }^{12,24}$ For the antiviral therapeutic area, sponsors should conduct a separate adolescent study in parallel with the adult phase 3 clinical trials if there are strong evidence of disease similarity between adults 
and pediatrics, and similarity in dosing/PK in adults and adolescents. Adolescents should be part of the primary endpoint analysis if they enroll in the adult phase 3 trial. Furthermore, the sponsor should begin development of a pediatric formulation as soon as the adult dose is selected from phase 2 trial(s). As more evidence becomes available, similar approaches may be possible for other indications. ${ }^{25}$

Before BPCA and PREA, more than $80 \%$ of the drugs approved for adult use were being used off-label in children. This number has been reduced to about $50 \%$ after passage of these laws but varies considerably depending on inpatient and outpatient settings. Based on our analysis since 2012, 85.2\% (46/54) of total studies for combined pediatric and adult trials were triggered by PREA, $11.1 \%(6 / 54)$ by BPCA, and $3.7 \%(2 / 54)$ by both for products reviewed under PREA and BPCA.

Enrollment of pediatric patients in adult trials was based on disease-specific criteria. When looking at disease-specific areas, cystic fibrosis and asthma were the primary diseases in the pulmonology area in adult and pediatric combined trials under BPCA/PREA. The large percentage of cystic fibrosis patients who are still in their teenage years facilitated pediatric and adult combined trials in the pulmonology area.

Subgroup analyses are often used to evaluate the efficacy and safety data in the trial across age subgroups. ${ }^{24}$ Approximately $51 \%$ of the combined studies reported a separate subanalysis for efficacy in pediatric patients, and only $42 \%$ reported a separate subanalysis for safety. These subgroup analyses are not required and may be impossible to do when the number of pediatric patients is small. Moreover, such subgroups are generally not powered sufficiently to provide independent substantiation of the efficacy or safety for that subgroup. Prior consideration of these subgroup analyses should be made during discussions between the sponsor and the FDA.

Combined adolescent-adult clinical trials were primarily conducted in phase 2 and 3 (Table S1) with a small percentage in phase 1 related to pharmacokinetics. The ACCELERATE trial, based on a literature review and multidisciplinary discussions between representatives from different fields, suggested that enrollment of adolescents of 12 years and over in adult early-phase oncology clinical drug trials, even in phase 1 first-in-human trials, may represent a safe and more efficient alternative method of obtaining a pediatric indication. ${ }^{26}$ Despite concerns of toxicity in pediatric oncology patients, the evidence to date appears to show that this approach is safe.

The U.S. FDA has granted many orphan drug designations since the introduction of the Orphan Drug Act in 1983. Nearly 4,000 drugs received orphan drug designations in the United States from 1983 to 2017, and more than 650 were approved for marketing during that time. ${ }^{27}$ The primary difference between the combined pediatric and adult trials under BPCA and/or PREA versus those under the Orphan Drug Act was that the ODA often included the entire pediatric age range in the trial. For $71 \%$ (32/45) of products, the entire pediatric age range was considered for the ODA trials, and this is approximately what was observed in a much broader analysis of the pediatric ODA products from a 20 year time period. ${ }^{11}$ In that FDA report to Congress, 221 of 348 indications (64\%) were considered to 
be fully labeled for pediatric use. These observations contrast with BPCA/PREA, where only 10 of 27 products (37\%) included combined trials with the entire pediatric age-range. Additional pediatric studies beyond the combined trials leading to a labeled pediatric indication in a younger population had to be conducted for 6 products under BPCA/PREA and 4 products under ODA in this analysis.

The therapeutic area with the greatest number of drugs with orphan designations and approvals is oncology. Oncology has the largest orphan approval number compared to other therapeutic areas from 2013 to 2017. ${ }^{28}$ This high number of orphan drugs in development for cancer is due to the ability to identify and target the underlying oncogenic driver events in subpopulations of cancer patients, and efforts to molecularly characterize hundreds of cancers from many oncology indications. ${ }^{29}$ When evaluating therapeutic classes, most orphan drug programs for the combined pediatric and adult trials focused on the hematology area. This may be related to age-specific incidence of lymphoma and leukemia among the pediatric population, ${ }^{23}$ with the most common cancers being lymphomas among adolescents 15 to 19 years old and leukemias in the 0 to 14 years of age group. ${ }^{30}$

One limitation to this study was that only publicly available information was used for this analysis. This approach was considered appropriate since most of the findings were objective (e.g. concurrent drug approval), and it was assumed that any subgroup analysis of safety and efficacy in pediatric patients would be considered sufficiently important to include in the publicly available review. Another limitation comes from the arbitrary nature of assigning age cut offs, as opposed to physiological criteria, in order to define what constitutes a pediatric patient, adolescent, or young adult. The 18 year old cutoff has been described as a hurdle for pediatric oncology patients. ${ }^{31}$ In addition, the physiologic development, disease pathophysiology, and drug pharmacology are other factors that may be considered when determining appropriate age cutoff criteria in studies. However, the ICH E11 age definitions used in this analysis ${ }^{17}$ are widely used by clinicians, drug developers and regulators.

In summary, pediatric drug approvals have been delayed in the past, with some products being labeled in pediatric patients more than 10 years after the adult drug approval. This study demonstrated that the use of combined pediatric and adult clinical drug development trials can result in approval for some or all pediatric populations at the same time as adult approval. Hematology had the largest percentage of drug approvals for combined pediatric and adult trials for drug and biological orphan products. Pulmonology was the only therapeutic area with many combined trials under BPCA and/or PREA. Most combined trials for products under BPCA/PREA were conducted using the adolescent patient population, in contrast to ODA combined trials that primarily used the entire pediatric age range. Approximately $50 \%$ of combined trials reported a separate subanalysis in pediatric patients for efficacy, and about $40 \%$ reported a separate subanalysis for safety. But the majority of the sub analyses were exploratory analyses and were not powered to independently confirm outcomes in the subgroup. Combined pediatric and adult drug development trials have been very successful at obtaining concurrent drug approval for part of the pediatric population at the time of the adult approval. 


\section{Supplementary Material}

Refer to Web version on PubMed Central for supplementary material.

\section{Acknowledgment}

We thank Clara Kim, University of Southern California, and Yiwei Yin, University of Michigan, for assisting with data collection.

Funding: No funding was received for this work.

\section{References}

1. Frattarelli DA, et al. Off-label use of drugs in children. Pediatrics 133, 563-567 (2014). [PubMed: 24567009]

2. Hwang TJ, Orenstein L, Kesselheim AS \& Bourgeois FT Completion Rate and Reporting of Mandatory Pediatric Postmarketing Studies Under the US Pediatric Research Equity Act. JAMA Pediatr 173, 68-74 (2019). [PubMed: 30452498]

3. Balan S, Hassali MAA \& Mak VSL Two decades of off-label prescribing in children: a literature review. World J Pediatr 14, 528-540 (2018). [PubMed: 30218415]

4. Pratico AD, et al. Off-Label Use of Drugs and Adverse Drug Reactions in Pediatric Units: A Prospective, Multicenter Study. Curr Drug Saf 13, 200-207 (2018). [PubMed: 29921210]

5. Tsukamoto K, et al. Improvement of Pediatric Drug Development: Regulatory and Practical Frameworks. Clin Ther 38, 574-581 (2016). [PubMed: 26869192]

6. Hudgins JD, Bacho MA, Olsen KL \& Bourgeois FT Pediatric drug information available at the time of new drug approvals: A cross-sectional analysis. Pharmacoepidemiol Drug Saf 27, 161-167 (2018). [PubMed: 29148107]

7. Sinha MS, Najafzadeh M, Rajasingh EK, Love J \& Kesselheim AS Labeling Changes and Costs for Clinical Trials Performed Under the US Food and Drug Administration Pediatric Exclusivity Extension, 2007 to 2012. JAMA Intern Med 178, 1458-1466 (2018). [PubMed: 30264138]

8. Wharton GT, et al. Impact of pediatric exclusivity on drug labeling and demonstrations of efficacy. Pediatrics 134, e512-518 (2014). [PubMed: 25022732]

9. US Food \& Drug Administration: Post-market requirements and commitments search. Access at: https://www.accessdata.fda.gov/scripts/cder/pmc/index.cfm (accessed on February 24, 2020) (2019).

10. Brennan Z FDA Closes Loophole Companies Used to Skirt Pediatric Study Requirements. Access at: https://www.raps.org/regulatory-focus\%E2\%84\%A2/news-articles/2017/12/fda-closesloophole-companies-used-to-skirt-pediatric-study-requirements (accessed on February 24, 2020) (2017).

11. US Food and Drug Administration: Pediatric Labeling of Orphan Drugs: Report to Congress. Access at: https://www.fda.gov/media/130060/download (accessed on February 24, 2020) (2019).

12. US Food and Drug Administration: Guidance for Industry: Considerations for the inclusion of adolescent patients in adult oncology clinical trials. Access at: https://www.fda.gov/regulatoryinformation/search-fda-guidance-documents/considerations-inclusion-adolescent-patients-adultoncology-clinical-trials (accessed on Febriary 24, 2020) (2019).

13. US Food and Drug Administration: Guidance for Industry: Pediatric HIV infection: drug development for treatment. Access at: https://www.fda.gov/regulatory-information/search-fdaguidance-documents/pediatric-hiv-infection-drug-development-treatment (accessed on February 24, 2020) (2019).

14. US Food and Drug Administration: Guidance for Industry: Demonstrating Substantial Evidence of Effectiveness for Human Drug and Biological Products. Access at: https://www.fda.gov/media/ 133660/download (accessed on April 12, 2020) (2019).

15. Green DJ, et al. Primary Endpoints in Pediatric Efficacy Trials Submitted to the US FDA. J Clin Pharmacol 58, 885-890 (2018). [PubMed: 29663424] 
16. Hudson MM Reproductive outcomes for survivors of childhood cancer. Obstet Gynecol 116, 11711183 (2010). [PubMed: 20966703]

17. US Food and Drug Administration: E11 Clinical Investigation of Medicinal Products in the Pediatric Population. Access at: https://www.fda.gov/regulatory-information/search-fda-guidancedocuments/e11-clinical-investigation-medicinal-products-pediatric-population (accessed on April 12, 2020) (2000).

18. Bogue C, et al. Special article: 2014 Pediatric Clinical Trials Forum. Pediatr Res 79, 662-669 (2016). [PubMed: 26650344]

19. European Medicines Agency: Reflection paper on the use of extrapolation in the development of medicines for paediatrics. Access at: https://www.ema.europa.eu/en/documents/scientificguideline/adopted-reflection-paper-use-extrapolation-development-medicines-paediatricsrevision-1_en.pdf (accessed on April 18, 2020) (2018).

20. Sun H, et al. Extrapolation of Efficacy in Pediatric Drug Development and Evidence-based Medicine: Progress and Lessons Learned. Ther Innov Regul Sci 2017, 1-7 (2017).

21. Chuk MK, Mulugeta Y, Roth-Cline M, Mehrotra N \& Reaman GH Enrolling Adolescents in Disease/Target-Appropriate Adult Oncology Clinical Trials of Investigational Agents. Clin Cancer Res 23, 9-12 (2017). [PubMed: 27780857]

22. Kapogiannis BG \& Mattison DR Adolescents in clinical trials. Clin Pharmacol Ther 84, 655-659 (2008). [PubMed: 19008903]

23. Mohamed MF, Rakhmanina N \& Hassan HE Inclusion of Adolescents With Adults in Phase 3 Clinical Trials: Overview of the Current State and a Call for Action. J Clin Pharmacol (2020).

24. DiGrande S AJMC Blog: FDA expands patient inclusion criteria for cancer clinical trials. Access at: https://www.ajmc.com/focus-of-the-week/fda-expands-patient-inclusion-criteria-for-cancerclinical-trials- (accessed on February 24, 2020) (2019).

25. Tanniou J, van der Tweel I, Teerenstra S \& Roes KC Subgroup analyses in confirmatory clinical trials: time to be specific about their purposes. BMC Med Res Methodol 16, 20 (2016). [PubMed: 26891992]

26. Gaspar N, et al. Joint adolescent-adult early phase clinical trials to improve access to new drugs for adolescents with cancer: proposals from the multi-stakeholder platform-ACCELERATE. Ann Oncol 29, 766-771 (2018). [PubMed: 29351570]

27. Redfearn S Tufts: Facing Many Challenges, Orphan Drugs Take 18\% Longer to Develop. Access at: https://www.centerwatch.com/cwweekly/2018/05/14/tufts-facing-many-challenges-orphandrugs-take-18-longer-to-develop (access on February 24, 2020) (2018).

28. Institute of Medicine: Safe and Effective Medicines for Children: Pediatric Studies Conducted Under the Best Pharmaceuticals for Children Act and the Pediatric Research Equity Act (2012), (The National Academies Press, Washington D.C., 2012).

29. Richon VM Drug discovery in rare indications: opportunities and challenges. Hematology Am Soc Hematol Educ Program 2013, 19-23 (2013). [PubMed: 24319157]

30. Steliarova-Foucher E, et al. International incidence of childhood cancer, 2001-10: a populationbased registry study. Lancet Oncol 18, 719-731 (2017). [PubMed: 28410997]

31. de Rojas T, et al. Access to Clinical Trials for Adolescents and Young Adults With Cancer: A Meta-Research Analysis. JNCI Cancer Spectrum 3, pkz057 (2019). [PubMed: 32337483] 


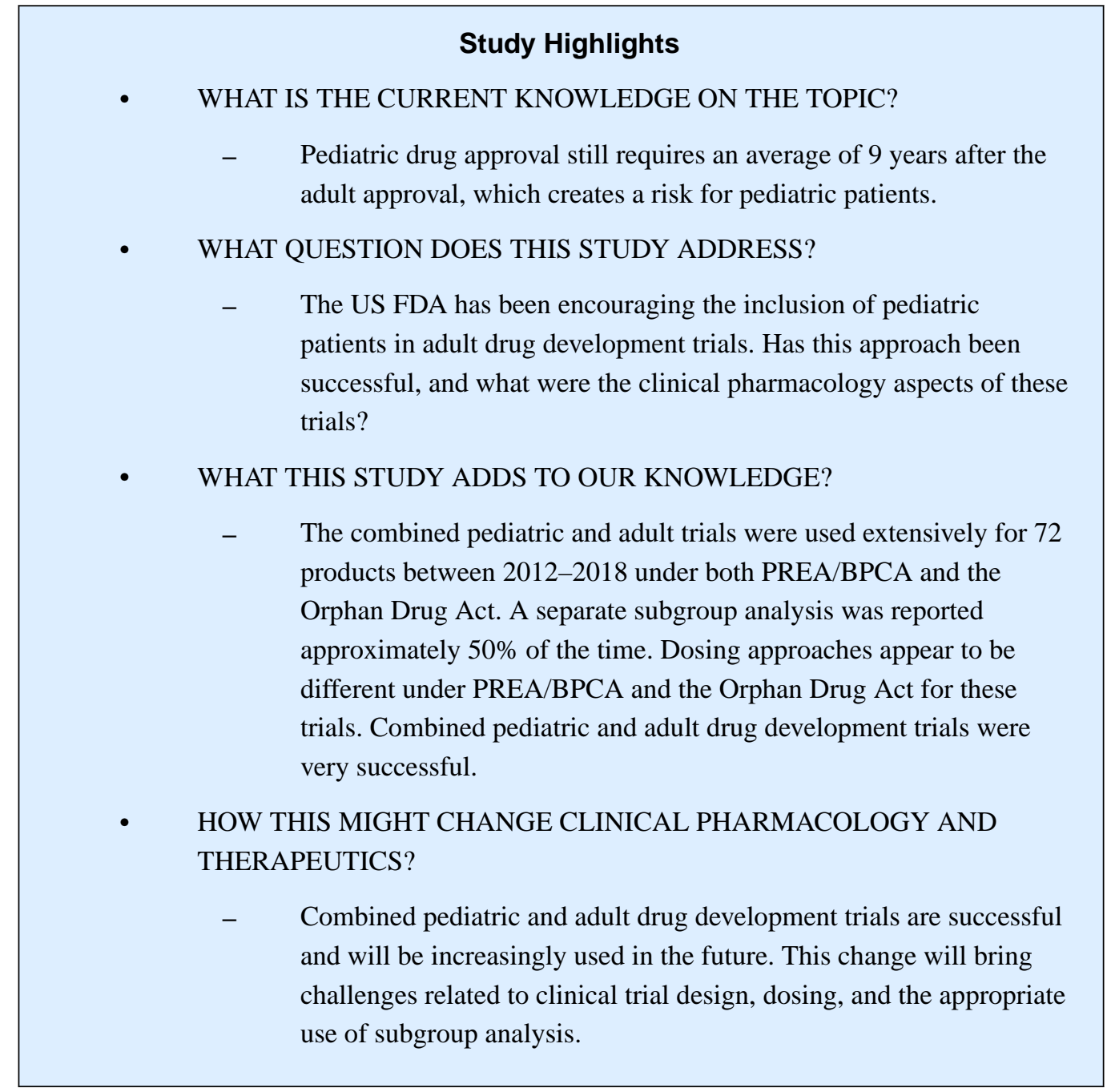


(a)

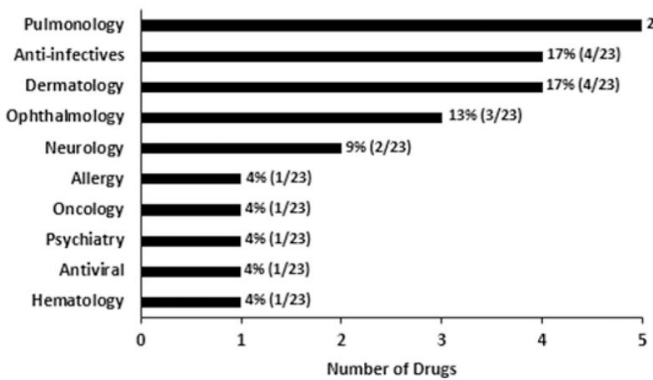

(c)

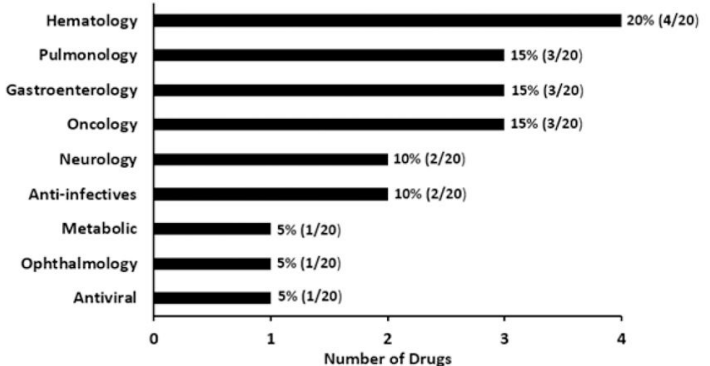

(b)

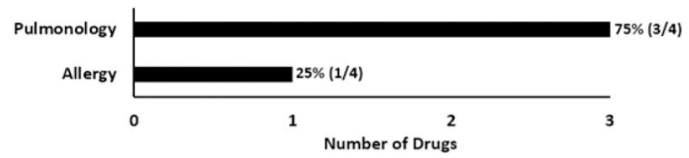

(d)

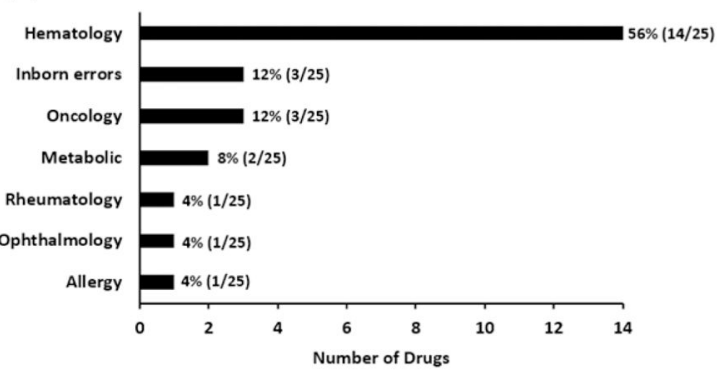

Figure 1.

Percentage and number of the approvals with pediatric patients included in adult trials based on total number of products from the years 2012-2018 under BPCA and/or PREA: a) drug products and b) biological products; under the Orphan Drug Act: c) drug and d) biological products. 
(a)

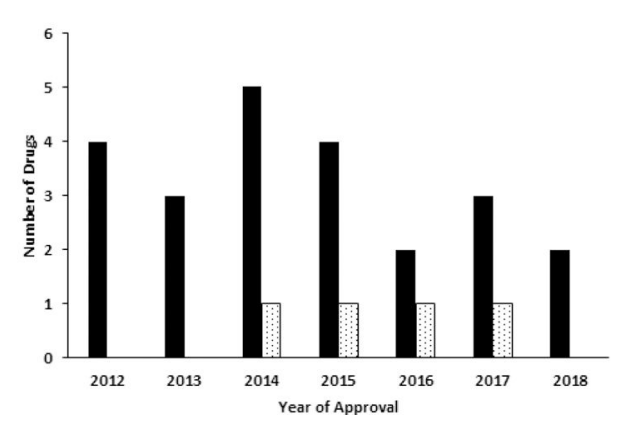

(b)

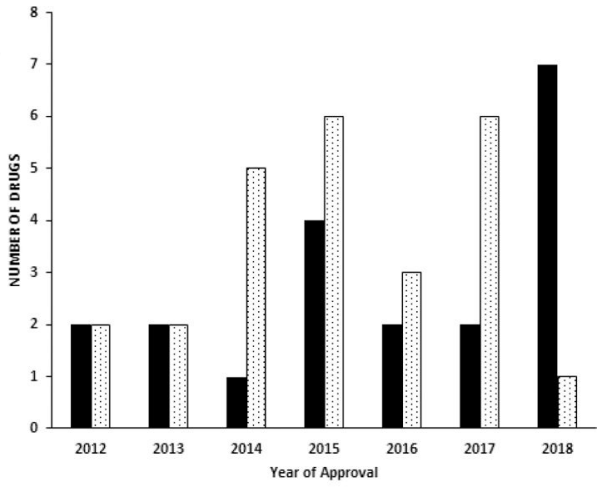

Figure 2.

Year of pediatric drug approval under a) BPCA and/or PREA, and b) the Orphan Drug Act with combined pediatric and adult trials. The black bars represent the drug products and the stippled bars represent the biological products. 
Table 1.

The age groups included in the combined adult and pediatric trials submitted to the FDA 2012-2018.

\begin{tabular}{|c|c|c|c|c|c|c|}
\hline \multirow[b]{2}{*}{ Age } & \multicolumn{3}{|c|}{ BPCA/PREA $^{a}$} & \multicolumn{3}{|c|}{ Orphan Drug Act } \\
\hline & $\begin{array}{l}\text { Number of } \\
\text { Products }\end{array}$ & $\begin{array}{c}\text { Number of } \\
\text { Trials }\end{array}$ & $\begin{array}{c}\text { Number of } \\
\text { Products with } \\
\text { Additional } \\
\text { Pediatric Studies }\end{array}$ & $\begin{array}{l}\text { Number of } \\
\text { Products }\end{array}$ & $\begin{array}{l}\text { Number of } \\
\text { Trials }\end{array}$ & $\begin{array}{l}\text { Number of Products } \\
\text { with Additional } \\
\text { Pediatric Studies }\end{array}$ \\
\hline $12-18$ years & 13 & 30 & 5 & 11 & 26 & 4 \\
\hline $6-18$ years & 4 & 7 & 1 & 2 & 2 & 0 \\
\hline All pediatric ${ }^{b}$ & 10 & 17 & 0 & 32 & 74 & 0 \\
\hline
\end{tabular}

${ }^{a}$ Abbreviations: BPCA, Best Pharmaceuticals for Children Act; PREA, Pediatric Research Equity Act.

${ }^{b}$ All pediatric includes birth to 18 years of age. 
Table 2.

Separate analysis for efficacy, safety, and dose finding for the pediatric subgroup in combined pediatric and adult trials reviewed under BPCA and/or PREA and the Orphan Drug Act.

\begin{tabular}{|c|l|c|c|c|}
\hline \multicolumn{1}{|c|}{ Regulatory Act } & Type of Products & $\begin{array}{c}\text { Separate Pediatric Efficacy } \\
\text { Analysis }\end{array}$ & $\begin{array}{c}\text { Separate Pediatric Safety } \\
\text { Analysis }\end{array}$ & Dose Finding Studies \\
\hline \multirow{2}{*}{$\mathrm{BPCA}^{a}$ and/or PREA } & Drug & $56.5 \%(26 / 46)$ & $45.7 \%(21 / 46)$ & $0 \%(0 / 46)$ \\
\cline { 2 - 5 } & Biologic & $62.5 \%(5 / 8)$ & $62.5 \%(5 / 8)$ & $12.5 \%(1 / 8)$ \\
\hline \multirow{2}{*}{ Orphan Drug Act } & Drug & $59.6 \%(28 / 47)$ & $44.7 \%(21 / 47)$ & $2.1 \%(1 / 47)$ \\
\cline { 2 - 5 } & Biologic & $38.2 \%(21 / 55)$ & $32.7 \%(18 / 55)$ & $7.3 \%(4 / 55)$ \\
\hline
\end{tabular}

${ }^{a}$ Abbreviations: BPCA, Best Pharmaceuticals for Children Act; PREA, Pediatric Research Equity Act. 
Table 3.

Different dosing approaches for combined pediatric and adult trials reviewed under BPCA and/or PREA and ODA for drug and biologic.

\begin{tabular}{|l|l|c|c|c|}
\hline Type of Drug Reviews & Type of Products & Fixed Dose & Weight Band & Weight Base \\
\hline \multirow{2}{*}{ BPCA and/or PREA } & Drug & $82.6 \%(38 / 46)$ & $6.5 \%(3 / 46)$ & $10.9 \%(5 / 46)$ \\
\cline { 2 - 5 } & Biologic & $87.5 \%(7 / 8)$ & $0 \%(0 / 8)$ & $12.5 \%(1 / 8)$ \\
\hline \multirow{2}{*}{ Orphan Drug Act } & Drug & $31.9 \%(15 / 47)^{a}$ & $38.3 \%(18 / 47)^{a}$ & $44.7 \%(21 / 47)^{a}$ \\
\cline { 2 - 5 } & Biologic & $30.9 \%(17 / 55)^{a}$ & $32.7 \%(18 / 55)^{a}$ & $83.6 \%(46 / 55)^{a}$ \\
\hline
\end{tabular}

${ }^{a}$ Some overlap between these dosing types 